\title{
Study on the harmonics overvoltage of large-scale photovoltaic power plant system based on passive network model
}

\author{
Chunlai $\mathrm{Li}^{1, \mathrm{a}}$, Jiacheng $\mathrm{Hao}^{2, \mathrm{~b}}$, Yongqiang $\mathrm{Zhu}^{2, \mathrm{c}}$, \\ Qi Tang ${ }^{2, d}$,Luming $\mathrm{Ge}^{3, \mathrm{e}}$ and Linan $\mathrm{Qu}^{3, \mathrm{f}}$ \\ ${ }^{1}$ Qinghai Province Key Laboratory of Photovoltaic Grid Connected Power Generation Technology, \\ Xining 810000, China \\ ${ }^{2}$ State Key Laboratory for Alternate Electrical Power System with Renewable Energy Sources, \\ North China Electric Power University, Beijing 102206 China \\ ${ }^{3}$ China Electric Power Research Institute, Nanjing 210003 China \\ alichunlai0216@163.com, ${ }^{b} 18811525108 @ 163 . c o m,{ }^{c} z y q @ n c e p u . e d u . c n,{ }^{d}$ tangqihd@163.com, \\ egeluming@epri.sgcc.com.cn, 'qulinan@epri.sgcc.com.cn
}

Keywords: Large-scale photovoltaic power station, Harmonic overvoltage, Passive network model, Electricity collection system.

Abstract. Harmonic problem is one of the main problems that restrict the development of grid connected photovoltaic (PV) power plant. In this paper, a passive network model of harmonic transmission is established with detailed consideration of the electricity collection system structure of PV power station based on the topological structure of typical large-scale grid connected PV power station. The harmonic power flow calculation is carried out by using the nodal admittance method in MATLAB. The magnification of the harmonic voltage of each node with respect to the fundamental wave voltage is concluded. The point of common coupling (PCC) is selected as the analysis object, and compared with the calculation results of the neglect of the electricity collection system, it is found that the characteristic of the harmonic voltage amplification is obviously changed. In the end, the influences of the LCL filter on the harmonic voltage amplification of the transmission network are analyzed.

\section{Introduction}

Large-scale PV power plant has become one of the important development trends of PV industry, and it is expected that by 2020, China's large-scale grid connected PV power plant will reach 80 million kilowatts[1].

In the large-scale PV power station, the output current of each group of grid connected inverter system is collected by the electricity collection system in the station and then sent out. One literature research shows that even if the output current harmonics of the single grid connected inverter are small, the output current harmonics of the multiple grid connected inverters are also likely to exceed the standard[2,3]. Harmonic problem is one of the main problems that restrict the development of grid connected PV power plant. Therefore, for large-scale PV power plants, it is necessary to consider the influence of the electricity collection system, which includes the filter, transformer and cable circuit and so on, on the harmonic transmission[4,5,6,7].

A mathematical model of harmonic series parallel resonance in large-scale PV power plant is proposed in Reference [8], and the mechanism of harmonic series parallel resonance and the relationships among the harmonic voltage amplification factor and the transmission distance, the frequency of harmonic and the type of harmonics are analyzed. But the internal components and their connection of electricity collection system of large-scale PV power station are not taken into account in Reference [8], and the influence of the power grid impedance on harmonic and resonance of large-scale PV power station is studied mainly from the inverter control level. Although the internal structure of PV power system is considered in detail in the analysis and calculation of Reference [9], the research content is the static voltage stability problem of PV power station, which is not related to 
the harmonic transmission network. In Reference [10], Thevenin equivalent circuit of 1MVA PV power generation is derived, an impedance network model is established which is close to the actual grid connected system, the relationship between the harmonic output of PV power station and irradiance and temperature of the PV panel is analyzed. But the influence of the parameters of each component in the model on the harmonic output is not discussed in Reference [10].

In this paper, a passive network model of harmonic transmission is established with detailed consideration of the electricity collection system structure of PV power station based on the topological structure of typical large-scale grid connected PV power station. The harmonic power flow calculation is carried out by using the nodal admittance method in MATLAB. The magnification of the harmonic voltage of each node with respect to the fundamental wave voltage is concluded. The PCC is selected as the analysis object, and compared with the calculation results of the neglect of the electricity collection system, it is found that the characteristic of the harmonic voltage amplification is obviously changed. In the end, the influences of the LCL filter on the harmonic voltage amplification of the transmission network are analyzed.

\section{Harmonic transmission network}

\section{Structure of large-scale grid connected PV power station}

Large-scale grid connected PV power plant structure is as shown in Figure 1. Large-scale PV power plant contains a number of PV power generation units, the output power of the PV power generation unit by the station imports into the $35 \mathrm{kV}$ AC bus. Assuming that there are a total of $m$ collection lines in parallel in the PV power station, each set of wire routing $\mathrm{PVV}$ power generation unit, and there are a total of $n$ PV power generation units in each collection line.The electric energy produced by PV power station, boosted by $35 / 121 \mathrm{kV}$ main transformer, is set into large-scale power grid through the high voltage transmission line.

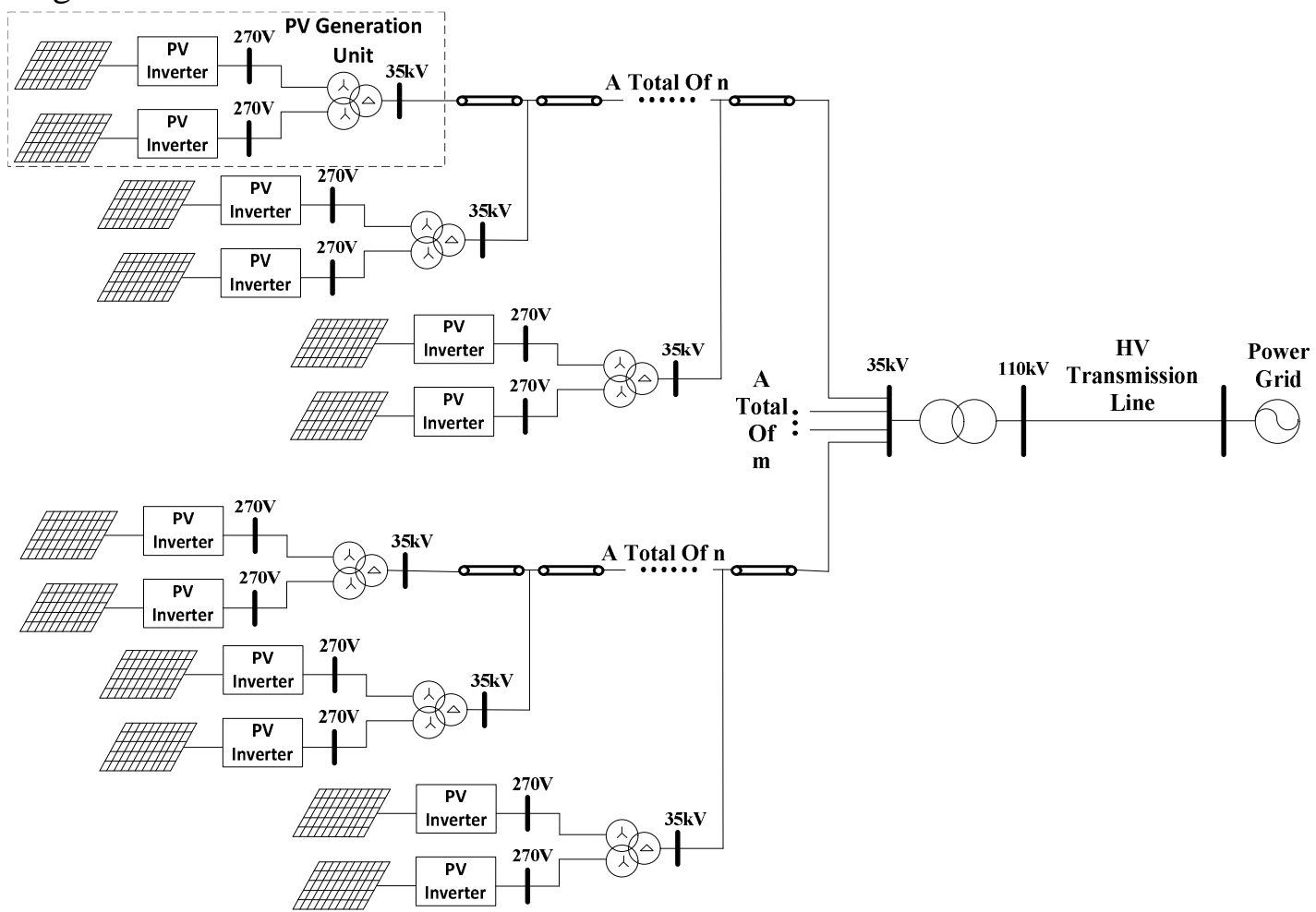

Fig. 1 The structure of large-scale PV power plant

The equivalent model of each component in the harmonic transmission network

PV power generation unit

In PV power generation unit, two sets of PV arrays are connected with two 500kW PV Inverters, the output current of the inverter is sent to a 35/0.27/0.27 kV double split transformer through the LCL filter and then access the station electricity collection system. 
The PV Inverter with current control mode can be equivalent to the constant current source in the harmonic power flow calculation.

The harmonic impedance of LCL filter can be expressed by formula 1, in which $h$ represents harmonic number.

$$
\left\{\begin{array}{l}
Z_{L}=j h X_{L} \\
Z_{c}=-j \frac{X_{c}}{h}
\end{array}\right.
$$

The short circuit impedance of $4.5 \%$, rated capacity of 1000/500/500 kVA double split transformer is used in PV power generation unit. $\mathrm{Z}_{\mathrm{t} 1}$ indicates that the equivalent impedance of the high voltage winding, $\mathrm{Z}_{\mathrm{t} 2}$ and $\mathrm{Z}_{\mathrm{t} 3}$ respectively represent the equivalent impedance of the two low voltage windings. In general, the equivalent impedance of the double split transformer can be expressed by formula 2, in which $\mathrm{K}_{\mathrm{F}}$ represents split coefficient.

$$
\left\{\begin{array} { l } 
{ X _ { t 1 } = ( 1 - \frac { K _ { F } } { 4 } ) X _ { 1 - 2 } } \\
{ X _ { t 2 } = X _ { t 3 } = \frac { 1 } { 2 } K _ { F } X _ { 1 - 2 } }
\end{array} \Rightarrow \left\{\begin{array}{l}
Z_{t 1}=j h X_{t 1} \\
Z_{t 2}=Z_{t 3}=j h X_{t 2}
\end{array}\right.\right.
$$

The same structure, parameter and control strategy are adopted in each group of grid connected inverter system in large-scale PV power plant, and the model of the booster transformer is the same[11]. Therefore, it is assumed that the parameters of each component of the power generating units in Figure 1 are all the same.

\section{Transmission line and cable}

In harmonic calculation, the transmission line is usually equivalent to the distributed parameter circuit[11]. In order to facilitate the calculation, the hyperbolic function is usually used to calculate the equivalent impedance as formula 3 , in which $h$ represents harmonic number.

$$
\left\{\begin{array}{l}
z_{o h}=r_{o h}+j h x_{1} \\
y_{o h}=j h b_{1}
\end{array}\right.
$$

Considering the skin effect, the unit length resistance of the line can be calculated by the following formula:

$$
\begin{cases}r_{o h}=r_{1}, & h=1 \\ r_{o h}=0.288 r_{1}+0.138 \sqrt{h r_{1}}, & h \neq 1\end{cases}
$$

The impedance and admittance value of the transmission line and the cable with the length of $l$ can be calculated by the formula 5 , in which $h$ represents harmonic number.

$$
\left\{\begin{array}{l}
Z_{L h}=Z_{C h} s h \gamma_{h} l \\
Y_{L h}=2 \cdot \frac{c h \gamma_{h} l-1}{Z_{C h} s h \gamma_{h} l}
\end{array}\right.
$$

$Z_{\mathrm{Ch}}$ and $\gamma_{\mathrm{h}}$ respectively represent characteristic impedance and propagation function of line.

$$
\left\{\begin{array}{l}
Z_{C h}=\sqrt{z_{o h} / y_{o h}} \\
\gamma_{h}=\sqrt{z_{o h} y_{o h}}
\end{array}\right.
$$

\section{Main transformer}

Under the action of higher harmonic, the skin effect and proximity effect of transformer windings become more significant, and the resistance value should be increased ${ }^{[12]}$. In this paper, the harmonic impedance model proposed by IEEE is used.

$$
Z_{T}=h^{1.15} R_{T}+h X_{T}
$$

\section{Power grid}

$\mathrm{S}$ and $\cos \varphi$ respectively represent power and power factor of power grid, equivalent impedance of power grid can be expressed by formula 8, in which $h$ represents harmonic number.

$$
Z=\sqrt{h} R+j h X
$$




\section{Model of harmonic transmission network}

Impedance diagram of the large-scale grid connected PV power plant based on Figure 1 is showed in Figure 2.

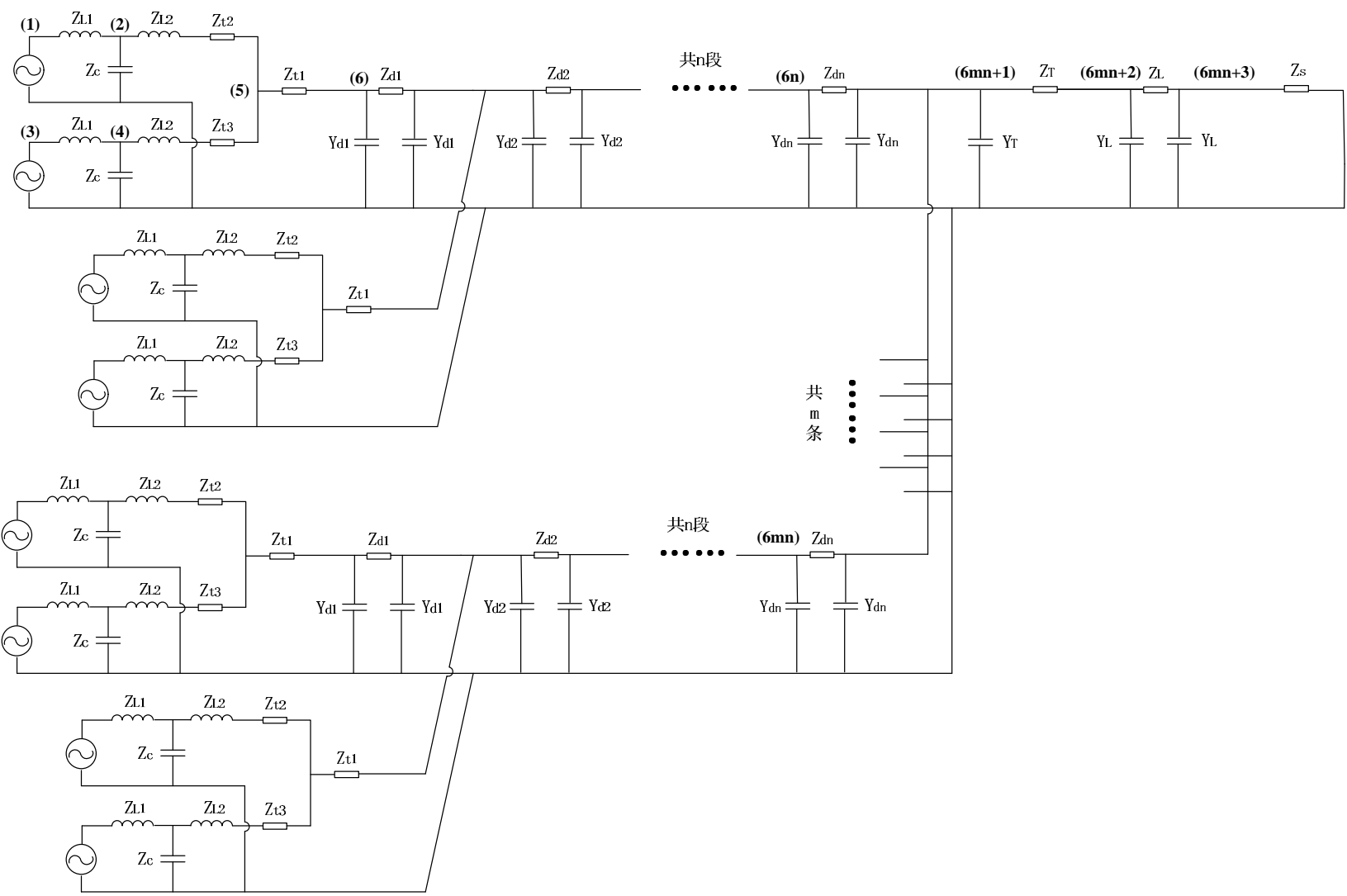

Fig. 2The equivalent impedance of large-scale PV power plant

According to the harmonic transfer network structure shown in Figure 2, we can get $\mathbf{Y}$, the nodal admittance matrix of the $(6 m n+3)$ order.

$$
\mathbf{Y}=\left[\begin{array}{ll}
\mathbf{A} & \mathbf{B} \\
\mathbf{C} & \mathbf{D}
\end{array}\right]
$$

A matrix is a (6mn)-order square matrix, $\mathbf{D}$ matrix is a 3-order square matrix, $\mathbf{A}$ matrix contains $m$ $(6 n)$-order square matrix $\mathbf{Y}_{\mathbf{m}}, \mathbf{Y}_{\mathbf{m}}$ matrix contains a (6n)-order square matrix $\mathbf{Y}_{\mathbf{1}}$ and (n-1) 6-order square matrix $\mathbf{Y}_{2}$.

$$
\begin{aligned}
& \mathbf{A}=\left[\begin{array}{llll}
\mathbf{Y}_{\mathrm{m}} & & & 0 \\
& \mathbf{Y}_{\mathrm{m}} & & \\
& & \mathrm{O} & \\
0 & & & \mathbf{Y}_{\mathrm{m}}
\end{array}\right] \\
& \mathbf{Y}_{\mathbf{m}}=\left[\begin{array}{llll}
\mathbf{Y}_{1} & & & \mathbf{0} \\
& \mathbf{Y}_{2} & & \\
& & \mathrm{O} & \\
\mathbf{0} & & & \mathbf{Y}_{2}
\end{array}\right]+\mathbf{Y}_{\mathrm{c}}
\end{aligned}
$$

$\mathbf{Y}_{1}$ represents the admittance relationship of the 6 nodes of the first power generation unit on a collection line, $\mathbf{Y}_{2}$ represents the admittance relationship of the 6 nodes of the remain power generation units on a collection line, $\mathbf{Y}_{\mathbf{c}}$, as a supplementary matrix, indicates the admittance relation among the power generating units. 


$$
\begin{array}{ll}
\mathbf{Y}_{1}(1,1)=\mathbf{Y}_{1}(3,3)=\frac{1}{Z_{L 1}} & \mathbf{Y}_{1}(2,2)=\mathbf{Y}_{1}(4,4)=\frac{1}{Z_{L 1}}+\frac{1}{Z_{C}}+\frac{1}{\left(Z_{L 2}+Z_{t 2}\right)} \\
\mathbf{Y}_{1}(5,5)=\frac{1}{\left(Z_{L 2}+Z_{t 2}\right)}+\frac{1}{\left(Z_{L 2}+Z_{t 3}\right)}+\frac{1}{Z_{t 1}} & \mathbf{Y}_{1}(6,6)=\frac{1}{Z_{t 1}}+Y_{d 1}+\frac{1}{Z_{d 1}} \\
\mathbf{Y}_{1}(1,2)=\mathbf{Y}_{1}(2,1)=\mathbf{Y}_{1}(3,4)=\mathbf{Y}_{1}(4,3)=-\frac{1}{Z_{L 1}} & \mathbf{Y}_{1}(2,5)=\mathbf{Y}_{1}(5,2)=-\frac{1}{\left(Z_{L 2}+Z_{t 2}\right)} \\
\mathbf{Y}_{1}(4,5)=\mathbf{Y}_{1}(5,4)=-\frac{1}{\left(Z_{L 2}+Z_{t 3}\right)} & \mathbf{Y}_{1}(5,6)=\mathbf{Y}_{1}(6,5)=-\frac{1}{Z_{t 1}}
\end{array}
$$

In addition to $\mathbf{Y}_{\mathbf{2}}(6,6)$, the rest of the $\mathbf{Y}_{\mathbf{2}}$ is the same as the $\mathbf{Y}_{\mathbf{1}}$. In the $i$-th power generation unit of the collection line:

$$
\mathbf{Y}_{2}(6,6)=\frac{1}{Z_{t 1}}+Y_{d(i-1)}+\frac{1}{Z_{d(i-1)}}+Y_{d i}+\frac{1}{Z_{d i}} \quad(i=2,3, \mathrm{~L}, n)
$$

$\mathbf{Y}_{\mathbf{c}}$ is the $(6 n)$-order symmetric matrix:

$$
\mathbf{Y}_{\mathbf{c}}(6 k, 6(k+1))=\mathbf{Y}_{\mathbf{c}}(6(k+1), 6 k)=-\frac{1}{Z_{d k}} \quad(k=1,2, \mathrm{~L}, n-1)
$$

$\mathbf{B}$ matrix and $\mathbf{C}$ matrix represent the admittance relationship between PCC and nodes connected to PCC. D matrix represents the nodal admittance relationship of nodes from PCC to power grid.

$$
\begin{array}{ll}
\mathbf{B}(6 n j, 1)=-\frac{1}{Z_{d n}} \quad(j=2,3, \mathrm{~L}, m) & \\
\mathbf{C}(1,6 n j)=-\frac{1}{Z_{d n}} \quad(j=2,3, \mathrm{~L}, m) & \\
\mathbf{D}(1,1)=\frac{m}{Z_{\mathrm{d} n}}+m \mathrm{Y}_{\mathrm{d} n}+\mathrm{Y}_{\mathrm{T}}+\frac{1}{Z_{\mathrm{T}}} & \mathbf{D}(2,2)=\frac{1}{Z_{\mathrm{L}}}+\mathrm{Y}_{\mathrm{L}}+\frac{1}{Z_{\mathrm{T}}} \\
\mathbf{D}(3,3)=\frac{1}{Z_{\mathrm{L}}}+\mathrm{Y}_{\mathrm{L}}+\frac{1}{Z_{\mathrm{S}}} & \mathbf{D}(1,2)=\mathbf{D}(2,1)=-\frac{1}{Z_{\mathrm{T}}} \\
\mathbf{D}(2,3)=\mathbf{D}(3,2)=-\frac{1}{Z_{\mathrm{L}}} &
\end{array}
$$

The parts that are not explained in the matrix $\mathbf{Y}$ are 0 .

\section{Analysis of Examples}

\section{Harmonic Flow Calculation}

Be similar to fundamental power flow calculation, harmonic flow calculation is analyzing the nodal voltage according to the injection harmonic current of each harmonic source and node admittance matrix of harmonic wave transfer network, which can be shown as follows:

$$
\mathbf{U}(h)=\mathbf{Y}^{-1}(h) \mathbf{I}(h)
$$

In this formula, $\mathbf{U}(h)$ means the column vector of $h$ times harmonic voltage of each node; $\mathbf{Y}(h)$ means the node admittance matrix of harmonic wave transfer network; $\mathbf{I}(h)$ means the column vector of injection $h$ times harmonic current. The injection current of each harmonic source is unit $h$ times harmonic current, the injection current of node without harmonic source is zero.

It should be noted that the equivalent impedance of each components in harmonic wave transfer network changes along with the order of harmonics. Therefore, the node admittance matrix of harmonic wave transfer network should be recalculated when the order of harmonics changes.

\section{Component Parameters of the Network}

In this paper, the rated capacity of PV power station is 20 MVA, about the electricity collection system, assuming $\mathrm{n}=5, \mathrm{~m}=4$. The rated capacity of the inverter is $500 \mathrm{~kW}$, the nominal voltage on the AC side is $270 \mathrm{~V}$. In the LCL filter, $\mathrm{L} 1=0.17 \mathrm{mH}, \mathrm{L} 2=0.05 \mathrm{mH}, \mathrm{C}=1900 \mu \mathrm{F}$. The rated capacity of double split transformer is $1000 / 500 / 500 \mathrm{kVA}$, ratio of transformation is $35 / 0.27 / 0.27 \mathrm{kV}, \mathrm{U}_{\mathrm{k}} \%=$ $4.5 \%$. The rated capacity of main transformer is $31.5 \mathrm{MVA}$, ratio of transformation is $121 / 35 \mathrm{kV}$, $\mathrm{P}_{0}=32.4 \mathrm{~kW}, \mathrm{P}_{\mathrm{k}}=140 \mathrm{~kW}, \mathrm{U}_{\mathrm{k}} \%=10.5 \%, \mathrm{I}_{0} \%=0.67 \%$. The type of cable is $\mathrm{YJV} 23-8.7 / 10,3 \times 185 \mathrm{~mm} 2$, the distance between adjacent PV generation units is $200 \mathrm{~m}$, the line impedance is $(0.128+\mathrm{j} 0.0913)$ 
$\Omega / \mathrm{km}$, and the line admittance is $1.194 \times 10-4 \mathrm{~S} / \mathrm{km}$. The type of overhead line is LGJ-400, the length of line is $150 \mathrm{~km}$, the line impedance is $(0.08+\mathrm{j} 0.391) \Omega / \mathrm{km}$, and the line admittance is $2.74 \times 10-6$ $\mathrm{S} / \mathrm{km}$. The minimum short-circuit capacity of power system is 2700 MVA, the power factor is 0.95 .

\section{Comparison of Harmonic Flow Calculation of Different Harmonic Wave Transfer Networks}

In the electricity collection system of large-scale PV power plant, because of short length and low voltage, the cable between adjacent PV generation units is always neglected in some studies and the PV generation units are simply in parallel.

After building up the harmonic wave transfer network, node voltages of different times harmonics can be calculated by harmonic flow calculation. Divide voltage magnitude of different times harmonics of one nodes by fundamental voltage magnitude of this node, you can get the voltage amplification factor of different times harmonics of this node. Because there are too much nodes in harmonic wave transfer network, this paper selects the node of low pressure side of main transformer, that is, the PCC of PV generation units, as an example.

Figure 3 shows the voltage amplification properties of PCC. As can be seen from the Figure, considering the parameters of cable in electricity collection system, the resonance point of network has shifted, the amplification factor of this point has decreased. The PCC voltage amplification properties of other times harmonics has also changed.

Thus it can be seen that to large-scale PV power plant, cable parameters of electricity collection system can cause certain influences to harmonics analysis results. So the parameters of cable should be considered when analyzing harmonics.

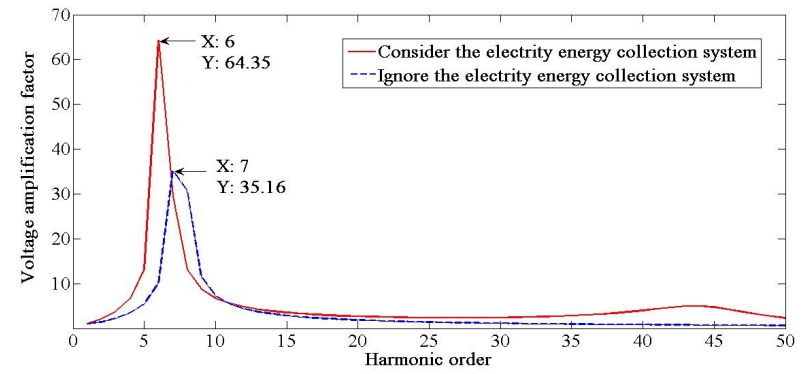

Fig. 3 The voltage amplification properties of PCC

The Effect of LCL Filter Parameters on Harmonic Flow Calculation Result

Considering the cable parameters of electricity collection system, based on the given parameters of all components, adjust the parameters of LCL filter $\left(L_{1}, L_{2}\right.$ and $\left.C\right)$ separately, the voltage amplification properties of PCC are shown in Figure 4-6.

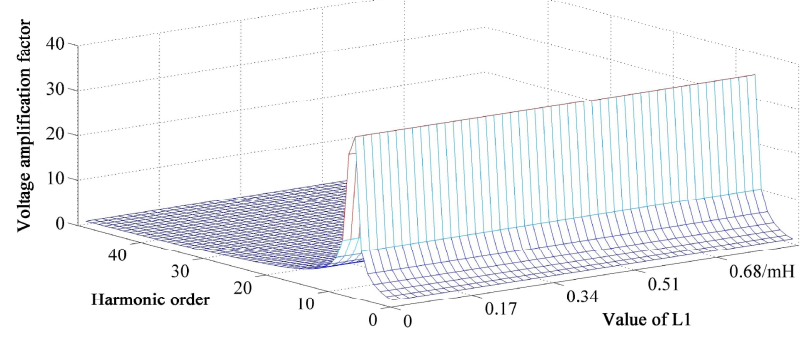

Fig. 4 The affection of $L_{1}$ to the voltage amplification properties of PCC

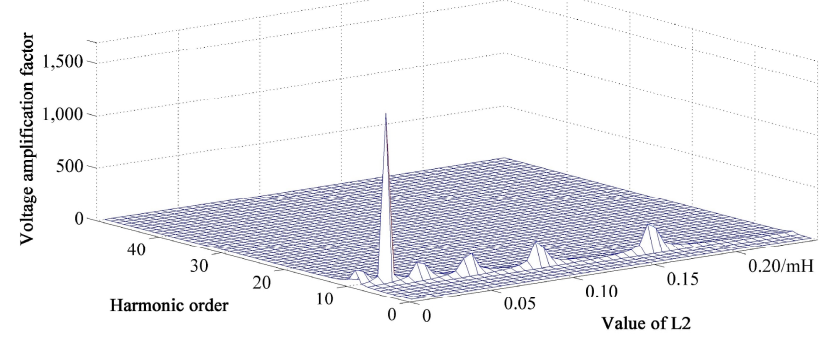

Fig. 5 The affection of $L_{2}$ to the voltage amplification properties of PCC 


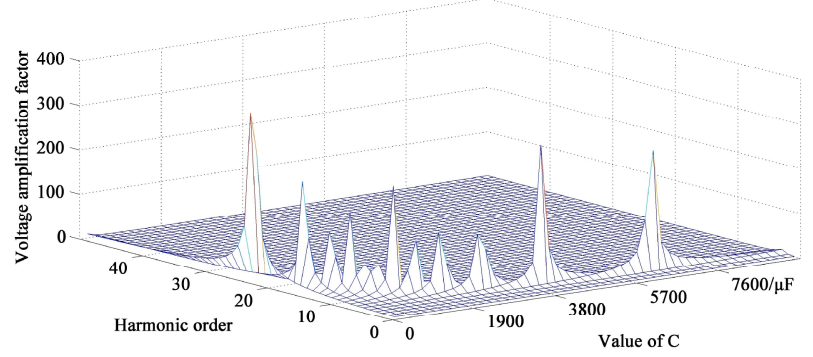

Fig. 6 The affection of $\mathrm{C}$ to the voltage amplification properties of PCC

From Figure 4-6, the changes of $L_{1}$ has no obvious influence on the voltage amplification properties of PCC. But the possibility of its affection on other nodes cannot be ruled out. With the increasing of $L_{2}$, the resonant frequency of PCC decreases gradually. Similar with $L_{2}$, the resonant frequency of PCC decreases gradually with the increasing of $C$. Compared to $L_{2}$, the changes of $C$ can cause PCC resonance more easily. One thing to note here is that Figure 4-6 only represent the variation of the voltage amplification properties of PCC with the changes of parameters of LCL filter. It can be derived from figures that system resonance could be caused around resonance points in the figures above. When the parameter value interval becomes smaller, that is, do more experiments in the same range of variation of one parameter, some changes may be caused in the figures above. But no matter the parameter value interval is, the voltage amplification factors of one node at a certain frequency are same when we choose the same parameter value.

\section{Conclusion}

In this paper, a passive network model of harmonic transmission is established with detailed consideration of the collection system structure of PV power station based on the topological structure of typical large-scale grid connected PV power station. The harmonic power flow calculation is carried out by using the nodal admittance method in MATLAB. And results show:

(1) The collection system structure of PV power station has a significant influence on the harmonic voltage amplification characteristic of PCC.

(2) The harmonic voltage amplification characteristic of PCC is also influenced by LCL filter of PV inverter. And the influences of the changes of $L_{2}$ and $C$ value on the characteristic of harmonic voltage amplification are obvious while the effect of the change of $L_{1}$ on PCC is not obvious. But the possibility of its affection on other nodes in network cannot be ruled out.

In this paper, a passive network model of harmonic transmission is established with detailed consideration of the collection system structure of PV power station, which is advantageous to explain the mechanism and basic law of the harmonic voltage amplification of PV power station.

\section{References}

[1] Zhao Zhengming,Lei Yi,He Fanbo: Overview of large-scale grid-connected photovoltaic power plants (Automation of Electric Power Systems,2011,35(12): 101-107).

[2] Dong Weijie,Bai Xiaomin,Zhu Ninghui: Discussion on the power quality under grid-connection of intermittent power sources (Power System Technology,2013,37(5): 1265-1271).

[3] Varma R, Salama M: Large-scale photovoltaic solar power integration in transmission and distribution networks (Proceedings of IEEE power and energy society general meeting,Detroit,2011).

[4] Turner R, Walton S, Duke R: Stability and bandwidth implications of digitally controlled grid-connected parallel inverters (IEEE Transactions on Industrial Electronics,2010,57(11): 3685-3694).

[5] Yang Ming, Zhou Lin, Zhang Dongxia: Stability analysis of large-Scale photovoltaic power plants for the effect of grid impedance (Transaction of China Electro technical 
Society ,2013,28(9): 214-223).

[6] Mi, Ju Xiuli: Stability Analysis of Large-scale Photovoltaic Plants Due to Grid Impedances ( Proceedings of the CSEE,2013,34: 34-41+9).

[7] Zhou Lin, Zhang Mi: Analysis of Resonance Phenomenon in Large-Scale Photovoltaic Power Plant (Electric Power Automation Equipment,2014,06: 8-14).

[8] Xie Ning, Luo An,Ma Fujun: Harmonic Interaction Between Large-scale Photovoltaic Power Stations and Grid (Proceedings of the CSEE,2013,34: 9-16+4).

[9] Du Xiao,Zhou Lin,Guo Ke: Static Voltage Stability Analysis of Large-Scale Photovoltaic Plants (Power System Technology,2015,12: 3427-3434).

[10] Xie Ning, Luo An, Chen Yandong: Dynamic Modeling and Characteristic Analysis on Harmonics of Photovoltaic Power Stations ( Proceedings of the CSEE,2013,36: 10-17+4).

[11] Lu Jingjing: Power System Harmonic Analysis and Software Development (China Electric Power Research Institute,2015).

[12] George J. Wakileh: Power Systems Harmonics Fundamentals, Analysis and Filter Design (Beijing: China Machine Press,2010.10). 